\section{rev Psi}

Revista de Psicología (UNLP)

https://revistas.unlp.edu.ar/revpsi

\title{
Capital psicológico, pasión por el trabajo y comportamiento innovador: un estudio exploratorio con docentes santafesinos
}

\author{
Solana Salessi
}

Correspondencia

solanasalessi@gmail.com

Filiaciones institucionales

${ }^{1}$ Universidad Nacional de Rafaela (Argentina)

\section{Resumen}

El comportamiento laboral innovador constituye un conjunto de acciones tendientes a la exploración, generación, socialización y realización de ideas novedosas. Sus determinantes contextuales han sido establecidos por la literatura científica. No obstante, sus antecedentes disposicionales continuan siendo objeto de investigación. El objetivo de este estudio fue analizar el efecto directo e indirecto del capital psicológico y la pasión por el trabajo sobre el comportamiento innovador. Se diseñó un estudio empírico cuantitativo transversal. Una muestra de 458 docentes de nivel primario ( $96.4 \%$ mujeres; $\mathrm{M}_{\text {Edad }}=34.67$ años; $\mathrm{M}_{\text {antiguedad }}=7.77$ años) respondieron medidas estandarizadas de las variables. Análisis de mediación indicaron que la pasión armoniosa media parcialmente el efecto positivo del capital psicológico sobre las cuatro dimensiones del comportamiento innovador. Los resultados iluminan la importancia de desarrollar estados positivos en el trabajo como un medio de favorecer la innovación. Se señalan limitaciones del estudio realizado y líneas para la investigación futura.

\section{Palabras clave}

innovación pedagógica | capital psicológico | pasión armoniosa | mediación estadística

\section{Cómo citar}

Salessi, S. (2020). Capital psicológico, pasión por el trabajo y comportamiento innovador: un estudio exploratorio con docentes santafesinos. Revista de Psicología, 19(1), 88-103. doi: 10.24215/2422572XE045
29 feb. 2020

\section{Editor}

Nicolás Alessandroni | Facultad de Psicología, Universidad Autónoma de Madrid (España)
ISSN

2422-572X

Licencia

(c) Copyright: Salessi, S. Licencia de Cultura Libre CC-BY 4.0

Entidad editora

RevPsi es una publicación de la

Facultad de Psicología (Universidad Nacional de La Plata, Argentina)

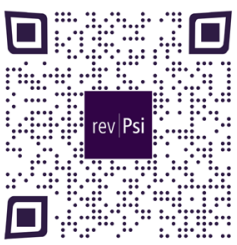

ACCESO ABIERTO DIAMANTE $\odot$ 


\title{
Capital psicológico, paixão pelo trabalho e comportamento inovador
}

\section{Resumo}

O comportamento inovador no trabalho constitui um conjunto de ações de exploração, geração, socialização e realização de novas ideias. Seus determinantes contextuais foram estabelecidos pela literatura científica. No entanto, suas raízes deposicionais continuam sendo objeto de investigação. O objetivo deste estudo foi analisar o efeito direto e indireto do capital psicológico e da paixão pelo trabalho no comportamento inovador. Foi realizado um estudo empírico transversal. Uma amostra de 458 professores das escolas primárias (96.4\% mulheres, $\mathrm{X}_{\text {edade }}=34.67$ anos, $\mathrm{X}_{\text {antiguidade }}=7.77$ anos) respondeu a medidas padronizadas das variáveis. Análises de mediação indicaram que a paixão harmoniosa medeia parcialmente o efeito positivo do capital psicológico nas quatro dimensões do comportamento inovador. Os resultados mostram a importância de desenvolver estados positivos no trabalho como forma de promover a inovação. Limitações do estudo realizado e linhas para pesquisas futuras são indicadas.

\section{Palavras-chave}

inovação pedagógica | capital psicológico | paixão pelo trabalho | mediação estatística

\section{Psychological capital, work passion and innovative behavior}

\begin{abstract}
The innovative work behaviour constitutes a set of actions such as opportunities exploration, idea generation, idea promotion and idea realization. Its contextual determinants have been established by scientific literature. However, its dispositional roots remain the subject of investigation. The objective of this study was to analyse the direct and indirect effect of psychological capital and work passion on innovative work behaviour. A cross-sectional empirical study was designed. A sample of 458 primary schools' teachers ( $96.4 \%$ women, $\mathrm{X}_{\text {age }}=34.67$ years, $\mathrm{X}_{\text {seniority }}=7.77$ years) answered standardized measures of the variables. Mediation analyses indicated that harmonious passion partially mediates the positive effect of psychological capital on the four dimensions of innovative behaviour. The results illuminate the importance of developing positive states at work as a way of promoting innovation. Limitations of the study carried out and lines for future research are indicated.
\end{abstract}

\section{Keywords}

pedagogical innovation | psychological capital | work passion | statistic mediation 


\section{Aspectos destacados del trabajo}

- El estudio se focaliza en una población poco explorada en la literatura sobre comportamiento innovador.

- Presenta evidencias empíricas sobre determinantes disposicionales del comportamiento laboral innovador.

- Analiza el interjuego entre variables novedosas dentro de la literatura organizacional.

- Sugiere la relevancia de desarrollar recursos y estados positivos para promover la innovación.

En un escenario social signado por la complejidad y la celeridad de los cambios, la innovación se ha posicionado como una cuestión estratégica para todos los actores sociales, entre los cuales la escuela no es una excepción. Aplicada al ámbito educativo, la innovación supone transformar positivamente aspectos o procesos curriculares o pedagógicos, con miras a lograr mayor calidad en los aprendizajes de los estudiantes (Organización de las Naciones Unidas para la Educación, la Ciencias y la Cultura, UNESCO, 2016). El presente estudio se focaliza en las innovaciones pedagógicas (Messmann, Stoffers, van der Heijden y Mulder, 2017), originadas a partir de la iniciativa del docente más allá de los requerimientos formales de su rol, y desarrolladas en el contexto de su práctica. Asumiendo al docente y a su comportamiento como unidad de análisis, adopta el término comportamiento laboral innovador (del inglés innovative work behaviour) para describir los esfuerzos orientados a crear, proponer y desarrollar cambios y mejoras positivas (Salessi y Omar, 2017).

La literatura académica concibe al comportamiento innovador como el conjunto de acciones tendientes a la generación, promoción y realización de ideas novedosas que pretenden introducir cambios positivos (De Spiegelaere, Van Gyes y Van Hootegem, 2014; Oeij, Rus y Pot, 2017). La innovación comienza con la concepción de una idea útil, original o novedosa que pretende responder las incongruencias o problemáticas percibidas en el entorno de trabajo; es decir, con la generación de ideas. La siguiente tarea consiste en la socialización de dicha idea entre los potenciales aliados. Vale decir, en la promoción de la idea novedosa con miras a obtener patrocinadores y construir las coaliciones necesarias para apoyarla. Finalmente, el proceso innovador concluye con la concreción de la idea novedosa mediante el diseño, producción y aplicación de un prototipo o modelo susceptible de experimentarse y evaluarse; esto es, con el desarrollo y realización de la idea (Salessi, en prensa).

Aunque el grueso de la investigación en la temática se ha focalizado en estudiar la incorporación de tecnologías de la información y la comunicación como soporte 
didáctico (Vu, Fredrickson y Moore, 2017); el comportamiento innovador docente trasciende ampliamente la mera innovación tecnológica. En este sentido, por ejemplo, es posible identificar innovaciones referidas a la disposición de los espacios escolares, la periodización del tiempo y el uso de instrumentos pedagógicos; innovaciones relativas a contenidos del currículo, métodos pedagógicos, estrategias de enseñanza, $\mathrm{y}$ actividades de aprendizaje; e innovaciones atinentes a roles, relaciones, objetivos, valores y fines. En última instancia, no se trata de una invención en sentido estricto, sino de una invención relativa a un contexto singular, donde una nueva práctica viene a producir una ruptura con las prácticas rutinarias que hasta el momento caracterizaban ese espacio (Messmer et al., 2017).

La evidencia reunida hasta el momento muestra que, además de factores situacionales tales como el apoyo organizacional, el clima de innovación y el estilo de liderazgo del director (Wang, Fang, Qureshi y Janssen, 2015), las diferencias individuales también podrían explicar porciones significativas de su varianza. En este sentido, estudios preliminares (Kaur y Gupta, 2016; Thurlings, Evers y Vermeulen, 2015) indican el impacto positivo de algunos rasgos de personalidad como la apertura a la experiencia, curiosidad, perseverancia, y la autoestima; de determinadas competencias (resolución de problemas, formación profesional y pedagógica, etc.); y de ciertas actitudes, tales como el compromiso organizacional y la satisfacción laboral.

La tendencia vigente en las investigaciones sobre la temática se orienta a explorar el potencial explicativo de otras variables disposicionales. Tal es el caso del capital psicológico o CapPsi (Luthans y Youssef-Morgan, 2017). En efecto, distintos autores (Abbas y Raja, 2015; Klaeijsen, Vermeulen y Martens, 2018; Montani, Odoardi y Battistelli, 2014; Sameer, 2018; Thurlings et al., 2015; Sun y Huang, 2019) sugieren vinculaciones significativas entre los recursos psicológicos de los empleados y su conducta innovadora. Asimismo, recientemente se ha postulado (Narang y Bhatnagar, 2017) que ciertos estados positivos podrían intervenir como mecanismos explicativos de diversos comportamientos proactivos, de los cuales la conducta innovadora constituye una variante específica. Con base en tales antecedentes, el objetivo de esta investigación fue analizar las relaciones directas e indirectas entre el CapPsi, la pasión por el trabajo y el comportamiento innovador en una muestra de docentes rafaelinas.

\section{Capital psicológico y comportamiento innovador}

El CapPsi designa una colección de recursos psíquicos individuales, que contribuye a la evaluación optimista de la probabilidad de éxito, basado en el esfuerzo y la perseverancia. Constituye un estado psicológico positivo caracterizado por tener confianza (autoeficacia) para asumir y llevar a cabo con éxito una tarea desafiante; hacer una atribución positiva (optimismo) sobre el resultado de la misma; perseverar en la búsqueda de los objetivos (esperanza); y fortalecerse en la adversidad (resiliencia) para alcanzar el éxito (Luthans y Youssef-Morgan, 2017). Se concibe como un estado 
abierto al cambio, que aun teniendo cierta estabilidad en el tiempo es susceptible de desarrollo mediante intervenciones de corta duración, destinadas a influir en cada una de sus dimensiones así como en el constructo nuclear (Carter y Youssef区Morgan, 2019).

Sus efectos motivacionales han demostrado ser más amplios e impactantes, en virtud de la interacción y sinergia entre sus componentes individuales. En términos generales, los estudios muestran resultados concordantes sobre el impacto positivo que el capital psicológico tiene sobre el rendimiento laboral (Carter y Youssef区Morgan, 2019; Luthans y Youssef-Morgan, 2017; Madrid, Diaz, Leka, Leiva y Barros, 2018). Partiendo de este cuerpo de evidencias, las investigaciones más recientes se han concentrado en explorar su influencia sobre el desempeño creativo y novedoso. En primer lugar, se ha demostrado que en comparación con sus cuatro componentes individuales, CapPsi predice mucho mejor el rendimiento creativo. Asimismo, se ha señalado, que este supracosntructo es un antecedente de soluciones más efectivas y de comportamientos originales frente a los problemas, tanto directamente como a través de otros mecanismo mediadores de naturaleza disposicional (Huang $y$ Luthans, 2015; Taştan, 2016). Del mismo modo, distintos estudios (Abbas y Raja, 2015; Klaeijsen et al., 2018; Montani et al., 2014; Sameer, 2018; Thurlings et al., 2015; Sun y Huang, 2019) han puntualizado que los empleados con mayor CapPsi son propensos a generar, promover e implementar exitosamente ideas innovadoras. Con base en tales referentes empíricos se postula que:

Hipótesis 1: el CapPsi se relacionará positivamente al comportamiento innovador.

\section{El rol mediador de la pasión por el trabajo}

La pasión por el trabajo se define como un estado de deseo persistente, basado en valoraciones cognitivas y afectivas del propio trabajo. Se trata de un estado positivo y significativo de bienestar derivado de las evaluaciones que el sujeto efectúa de su situación laboral, y que resulta en un trabajo consistente y constructivo (Salessi, Omar y Vaamonde, 2017). Siguiendo los postulados del modelo dualista (Vallerad, 2015), la internalización del trabajo en el self de modo libre y autónomo da origen a una pasión armoniosa y positiva, en la que el trabajo ocupa un espacio significativo pero no abrumador de la identidad.

La pasión armoniosa por el trabajo lleva a las personas a invertir esfuerzos sostenidos permitiendo desarrollar las propias fortalezas naturales y adquirir nuevas habilidades. Favorece sentimientos de competencia y eficacia personal, autonomía y emociones positivas; al tiempo que propicia el desarrollo de comportamientos positivos que van más allá de los requerimientos formales del rol (Salessi y Omar, 2017; Bañuelos, Descals y Castaño, 2016). Además de sus efectos directos sobre otras actitudes, conductas y resultados organizacionalmente relevantes, los especialistas (Bañuelos et al., 2016; Curran, Hill, Appleton, Vallerand y Standage, 2015; Narang y Bhatnagar, 2017; Vallerand, 2015) consideran que la pasión podría desempeñarse 
como "tercera variable"; vale decir, interviniendo en la fuerza y dirección de las asociaciones entre otras variables. En línea con esta novedosa agenda científica, la presente propuesta pretende examinar si la pasión de los docentes hacia su trabajo media las relaciones entre el capital psicológico y el comportamiento innovador.

El marco conceptual provisto por el modelo de ampliación y construcción emocional (Kok y Fredrickson, 2013) permite comprender más acabadamente este posible rol. De acuerdo con esta perspectiva teórica, los estados emocionales positivos incrementan los recursos psicológicos y amplifican las posibilidades de la atención, la cognición y la acción, traduciéndose esto en una espiral ascendente de emocionalidad positiva. La literatura internacional (Salessi et al., 2017; Bañuelos et al., 2016; Curran et al., 2015; Vallerand, 2015) coincide en resaltar que la pasión se asocia intrínsecamente a un estado emocional placentero y, como tal, a un factor motivacional positivo con efecto significativo sobre el comportamiento. Por lo tanto, el componente afectivo que conlleva podría potenciar los recursos psicológicos favoreciendo tanto la identificación de oportunidades de mejora y la generación de ideas novedosas, como la perseverancia y movilización de recursos necesaria para su efectiva implementación. Con base en estos antecedentes se conjetura que:

Hipótesis 2: la pasión armoniosa mediará las relaciones entre el CapPsi y el comportamiento innovador en el trabajo.

\section{Método}

\section{Diseño}

La presente investigación corresponde a un estudio empírico, cuantitativo y transversal. Se enmarca en los lineamientos de la estrategia asociativa-explicativa (Ato, López y Benavente, 2013), ya que su finalidad fue explorar la relación funcional existente entre variables.

\section{Participantes}

Se trabajó con una muestra integrada por 458 docentes de diversas escuelas de gestión pública y privada, radicadas en el nodo Rafaela, Santa Fe, y correspondientes a los niveles de educación inicial y primaria del Sistema Educativo Nacional Argentino. La muestra fue seleccionada en forma no probabilística, según un muestreo por disponibilidad. El $96.42 \%$ de la muestra fueron mujeres. La edad promedio de los participantes fue de 34.67 años $(\mathrm{DT}=8.84)$. La antigüedad laboral media fue de 7.77 años (DT $=7.88$ ). El $89.50 \%$ de la muestra se desempeñaba en organizaciones de gestión estatal, en tanto que el $56.62 \%$ pertenecían a instituciones de nivel primario.

\section{Instrumentos}

Los participantes completaron de manera individual un cuadernillo conteniendo en su primera hoja el objetivo del estudio y las instrucciones para responder; en la 
segunda hoja el formulario de consentimiento informado y; en las restantes páginas los ítems correspondientes a los instrumentos que a continuación se describen. Tales ítems fueron aleatoriamente distribuidos. El protocolo de recolección incluyó, además, una sección de características sociodemográficas a efectos de caracterizar a la muestra bajo estudio.

Escala de Comportamiento Innovador Docente (Salessi, en prensa). Se compone por 12 ítems distribuidos en cuatro sub-escalas: exploración de oportunidades (ej.: "Busco sistemáticamente nuevos métodos, técnicas o instrumentos de trabajo"; $\alpha=.86$ ); generación de ideas (ej.: "Diseño actividades novedosas para promover el aprendizaje de mis alumnos"; $\alpha=.87$ ); socialización de ideas y búsqueda de recursos (ej.: "Consigo los recursos necesarios para poder llevar a la práctica mis ideas"; $\alpha=.72$ ); y realización de ideas (ej.: "Implemento actividades originales para favorecer el aprendizaje"; $\alpha=.83$ ). Los ítems son presentados con un formato tipo Likert de frecuencia de 5 puntos ( $1=$ Nunca; $5=$ Siempre).

Escala de Capital Psicológico (Omar, Salessi y Urteaga, 2016). El instrumento está conformado por 16 ítems con formato de respuesta tipo Likert de 5 puntos ( $1=$ Nunca; $5=$ Siempre), que miden las cuatro facetas del CapPsi a razón de 4 ítems por escala: esperanza (ej.: "Tengo fe de que al fin mis asuntos laborales van a mejorar"; $\alpha=.77$ ); optimismo (ej.: "Miro el lado positivo de cada proyecto laboral que emprendo"; $\alpha=.82$ ); resiliencia (ej.: "Cuando tengo una dificultad en mi trabajo, la supero con éxito"; $\alpha=.80$ ), y autoeficacia (ej.: "Si me esfuerzo lo necesario, puedo resolver problemas difíciles en mi trabajo"; $\alpha=.88$ ).

Escala de Pasión Armoniosa por el Trabajo (Salessi y Omar, en prensa). Se encuentra integrada por 7 ítems (ej.: "Las cosas que aprendo en este trabajo hacen que lo valore más todavía"; $\alpha=.89)$ presentados en formato Likert de 5 puntos $(1=$ Totalmente en desacuerdo; $5=$ Totalmente de acuerdo).

\section{Procedimiento}

La recolección de los datos se efectuó en el seno de aquellas instituciones educativas que, luego de conocer los objetivos del estudio, aceptaron formar parte de la investigación. En todos los casos, los participantes completaron de manera individual los instrumentos, durante los horarios de trabajo y en los lugares físicos destinados por los directivos para tal propósito. No se brindaron incentivos de ningún tipo. La administración de las técnicas estuvo a cargo de personal entrenado a tal efecto. Su ejecución se llevó a cabo de acuerdo con los lineamientos éticos establecidos por la Asociación Americana de Psicología (2017) y las recomendaciones del CONICET para las investigaciones en las ciencias sociales y humanas (Resolución 2827/06). El tiempo aproximado para completar el cuestionario fue de 20 minutos. En cada caso se explicó previamente el objetivo del estudio, se explicitaron las instrucciones para responder, y se aseguró el anonimato y la confidencialidad de la información 
suministrada. Todos los sujetos que aceptaron participar voluntariamente del estudio firmaron el correspondiente consentimiento informado.

\section{Estrategia de análisis}

El procesamiento y análisis de los datos se realizó con auxilio del paquete SPSS (versión 22.0), el macro PROCESS (Hayes, 2013) y el programa $G^{*}$ Power.

Análisis exploratorios. Se examinaron los datos con miras a detectar la presencia de valores perdidos y puntuaciones extremas. El análisis de datos perdidos consistió, principalmente, en valorar su proporción y la posible presencia de sesgos en su distribución. Para ello, se aplicó el test conjunto de aleatoriedad de Little. Los casos atípicos se identificaron mediante el cálculo de puntuaciones $\mathrm{Z}$ y de las distancias de Mahalanobis al cuadrado. Se analizó la distribución de las variables mediante el cálculo de estadísticos descriptivos y coeficientes de asimetría y curtosis (Tabachnick y Fidell, 2013).

Evidencias de confiabilidad de las escalas utilizadas se establecieron a partir del computó del coeficiente de confiabilidad compuesta $(\mathrm{H})$ y del coeficiente Omega de McDonald $(\omega)$. En ambos índices, valores iguales o superiores a .70 son evidencia de adecuada confiabilidad (Domínguez-Lara, 2016). La validez convergentediscriminante, en tanto, se determinó mediante el cálculo de la varianza media extraída (AVE, del inglés Average Variance Extracted) y de su raíz cuadrada, respectivamente. El AVE permite estimar la varianza común entre los indicadores y su factor latente, considerando apropiados valores iguales o superiores a .50 . Asimismo, valores de su raíz cuadrada superiores a la correlación entre los factores latentes, se asume como evidencia de que cada constructo comparte más varianza con sus indicadores que con los demás (Cheung y Chang, 2017).

Finalmente, dado que todas las variables fueron medidas con instrumentos autodescriptivos, se consideró pertinente explorar si los datos se encontraban sesgados debido al método común. Con esta finalidad se ejecutó el test del factor único de Harman (Podsakoff, Mackenzie y Podsakoff, 2012).

Verificación empírica. Se ejecutaron análisis correlacionales entre todas las variables de interés incluyendo, además, las variables edad y antigüedad laboral para explorar posibles vinculaciones significativas. Complementariamente, se calcularon pruebas de diferencias de medias ( $t$ de Student) para el tipo de gestión (pública vs. privada) y el nivel educativo (inicia vs. primario). Estos cómputos se realizaron a fin de detectar posibles diferencias que pudieran ameritar el control de estas variables sociodemográficas.

Para contratar las hipótesis de trabajo se estimaron los efectos totales del CapPsi sobre el comportamiento innovador, determinando los coeficientes correspondientes a los efectos directos (sin mediación de la pasión por el trabajo), e indirectos y residuales (a través de la mediación de la pasión por el trabajo). Con tal propósito, se seleccionaron 
5000 repeticiones del conjunto de datos para estimar los coeficientes e intervalos de confianza al 95\% (Hayes, 2013). Finalmente, se obtuvieron indicadores para el porcentaje de varianza explicada y el tamaño de efecto a partir de los estadísticos $R^{2}$ y $f^{2}$ de Cohen, respectivamente (Tabachnick y Fidell, 2013).

\section{Resultados}

\section{Análisis exploratorios.}

El porcentaje de datos perdidos en cada ítem no superó el 5\%. La prueba de Little indicó que el patrón de valores perdidos respondía completamente al azar (MCAR; $\chi_{(144)}^{2}=$ 152.08, $p=.215)$. Estos datos fueron reemplazados matemáticamente por valores calculados a partir del método EM (expectación-maximización). No se detectaron problemas de asimetría y/o curtosis extrema, ni observaciones atípicas desde una perspectiva univariada o multivariada. Para todas las escalas se obtuvo evidencia de adecuada confiabilidad y validez. La prueba de un solo factor de Harman mostró que el porcentaje de varianza explicada por un solo factor era tan sólo del $12.41 \%$, por lo que los datos no se encontraban sesgados por el método común (Podsakoff et al., 2012). En la Tabla 1 se presentan los estadísticos descriptivos, los coeficientes de confiabilidad y validez, y las correlaciones bivariadas entre las variables estudiadas.

La inspección de la Tabla 1 muestra que todas las dimensiones del comportamiento innovador se relacionan positivamente con el CapPsi y con la pasión por el trabajo, con una leve primacía de la exploración de oportunidades sobre el resto de las facetas. Del mismo modo, se observan asociaciones significativas entre el CapPsi y la pasión armoniosa; en tanto que pequeñas correlaciones entre la edad, la antigüedad laboral y el resto de las variables analizadas. En cuanto a las pruebas de diferencias de medias, no se encontraron diferencias significativas ni para el tipo de gestión ni para el nivel educativo en ninguna de las dimensiones del comportamiento innovador.

\section{Verificación empírica}

La Tabla 2 presenta los coeficientes estandarizados correspondientes a los efectos totales, indirectos y directos residuales del CapPsi y la pasión por el trabajo sobre el comportamiento laboral innovador. De la observación de la Tabla 2 se desprende que la pasión emerge como un mediador parcial en las relaciones entre el CapPsi y el comportamiento innovador, desde el momento que los efectos directos residuales de los recursos psicológicos en presencia de la pasión por el trabajo continúan siendo estadísticamente significativos. En conjunto, el modelo explica entre el 13\% y el 21\% de la variabilidad del comportamiento innovador tratándose en todos los casos de efectos moderados pero significativos según lo indican los valores del estadístico $f^{2}$ de Cohen. Los resultados obtenidos brindan apoyo empírico a la hipótesis 1, que adelantaba vinculaciones positivas entre el CapPsi el comportamiento innovador; $y$ a la hipótesis 2, que postulaba el rol mediador de la pasión por el trabajo. 


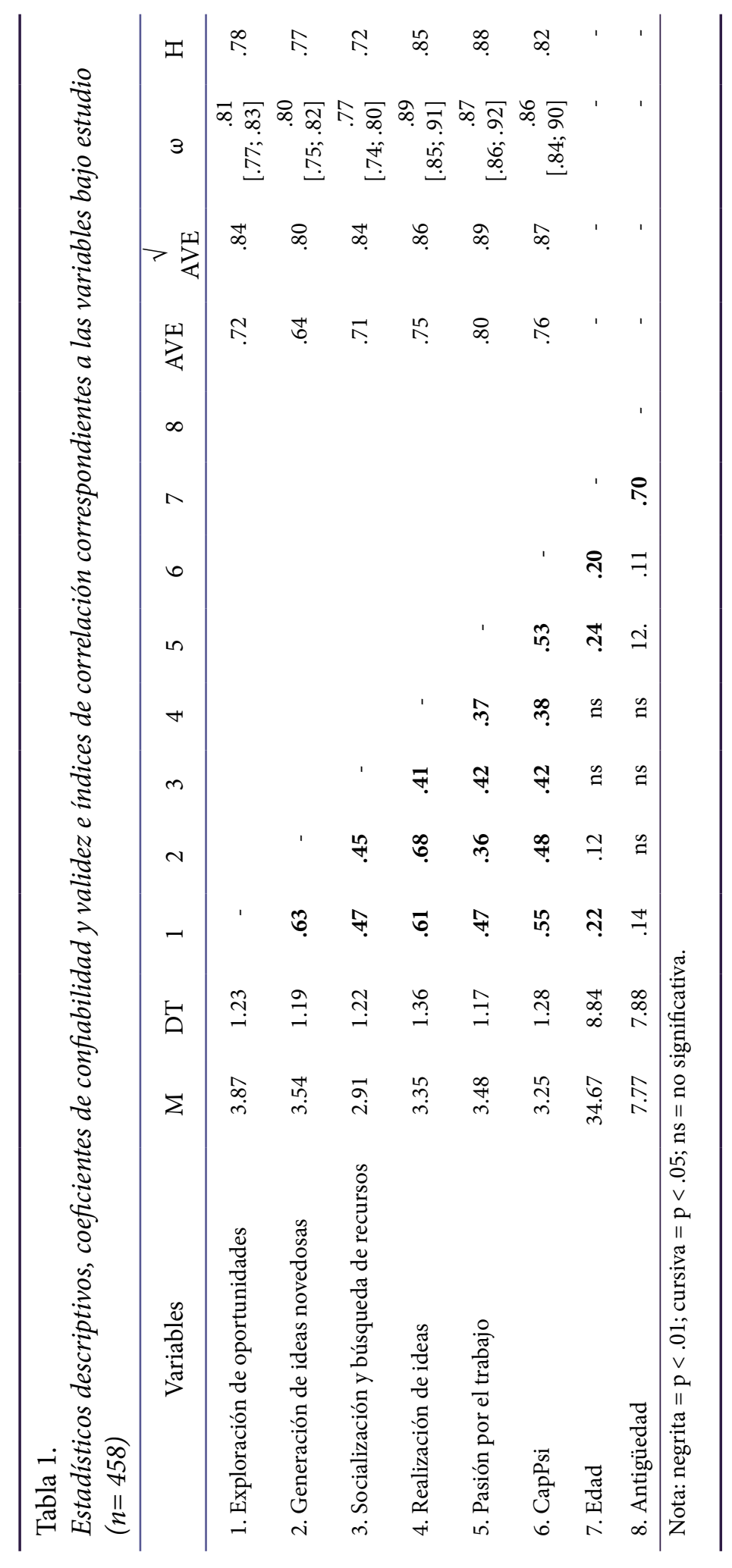


Tabla 2.

Efectos totales, indirectos y residuales del CapPsi sobre las dimensiones del comportamiento innovador

\begin{tabular}{|c|c|c|c|}
\hline Variables & $\begin{array}{l}\text { Efectos } \\
\text { totales }\end{array}$ & $\begin{array}{c}\text { Efectos } \\
\text { indirectos }\end{array}$ & $\begin{array}{c}\text { Edectos directos } \\
\text { residuales }\end{array}$ \\
\hline $\begin{array}{l}\text { CapPsi -> Exploración de } \\
\text { oportunidades }\end{array}$ & $.55^{\star *}$ & $.13^{* *}$ & $.42^{* *}$ \\
\hline CapPsi -> Generación de ideas & $.48^{\star *}$ & $.08^{* *}$ & $.40^{* *}$ \\
\hline CapPsi -> Socialización de ideas & $.42^{\star *}$ & $.15^{* *}$ & $.27^{* *}$ \\
\hline CapPsi -> Realización de ideas & $.38^{\star *}$ & $.12^{* *}$ & $.26^{* *}$ \\
\hline \multicolumn{4}{|c|}{$\begin{array}{l}\text { Nota: La flecha indica la dirección de la relación. Efectos totales }=\text { efectos indirectos }+ \text { efectos resi- } \\
\text { duales. Efectos indirectos= efectos del CapPsi a través de la pasión por el trabajo. Efectos directos } \\
\text { residuales }=\text { efectos directos del CapPsi. }{ }^{* \star}=\mathrm{p}<.001 .\end{array}$} \\
\hline
\end{tabular}

La Figura 1 informa los coeficientes beta estandarizados, los porcentajes de varianza explicada y el tamaño de efecto.

\section{Discusión}

La investigación en psicología organizacional contemporánea ya no se limita a estudios de primera generación, orientados al análisis de las relaciones directas entre las variables. Por el contrario, la tendencia actual se inscribe en las investigaciones de segunda generación; vale decir, estudios orientados a identificar terceras variables que medien o moderen el comportamiento de otras, explicando más acabadamente la intensidad y dirección de las vinculaciones subyacentes (Hopwood, 2007). El presente trabajo se encuadró, precisamente, en esta perspectiva, desde el momento que su objetivo fue verificar un modelo explicativo del comportamiento innovador que contempló efectos directos e indirectos del CapPsi y la pasión por el trabajo.

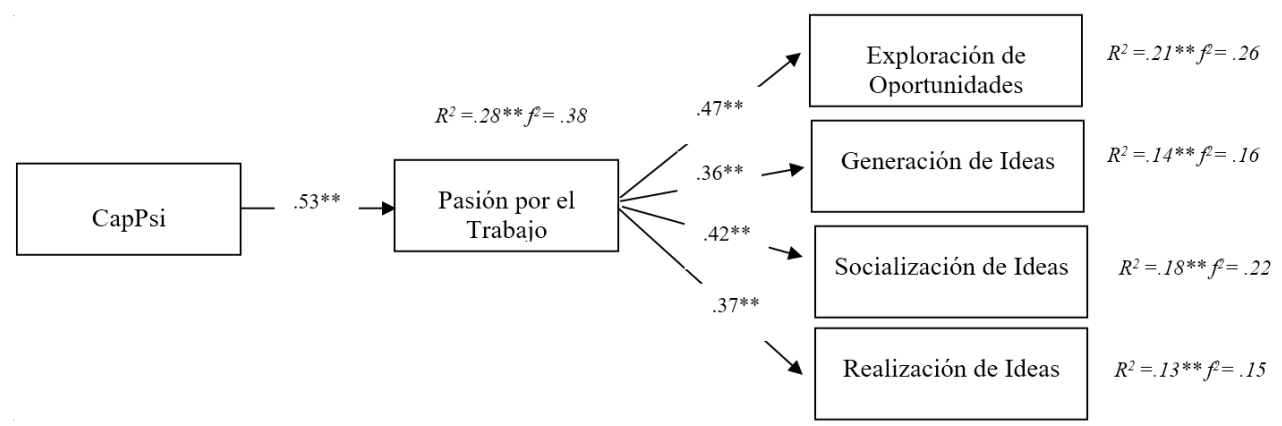

Figura 1. Coeficientes de regresión estimados, varianza explicada y tamaño de efecto correspondiente a las relaciones entre CapPsi, pasión por el trabajo y comportamiento laboral innovador (Fuente: elaboración propia). Nota: ${ }^{* *}=p<.01$. 
Los resultados obtenidos permiten una toma de posición frente al rol desempeñado por cada variable. En este sentido, además de ratificar las conexiones positivas entre el CapPsi y las cuatro dimensiones del comportamiento laboral innovador informadas en otros estudios (Abbas y Raja, 2015; Klaeijsen et al., 2018; Montani et al., 2014; Sameer, 2018; Thurlings et al., 2015; Sun y Huang, 2019), el presente trabajo demuestra la mediación parcial ejercida por la pasión armoniosa en tales relaciones.

En lo que hace a las vinculaciones directas entre el CapPsi y el comportamiento innovador, los hallazgos que se desprenden de este estudio exploratorio permiten concluir que aquellos empleados que poseen altos recursos psicológicos son propensos a generar, promover e implementar exitosamente ideas creativas y novedosas en su trabajo. La interacción y sinergia entre sus componentes podría explicar su efecto positivo sobre el comportamiento (Luthans y Youssef-Morgan, 2017). En este sentido, la autoeficacia generaría confianza en las propias capacidades para movilizar los recursos o cursos de acción necesarios para desarrollar la idea innovadora. El optimismo, en tanto, promovería una perspectiva de resultados positivos, así como la motivación suficiente para persuadir a los demás de la importancia de la idea. La esperanza favorecería la voluntad de alcanzar la meta propuesta, y la perseverancia y determinación necesaria para lograrla. Finalmente, la resiliencia impulsaría a recuperarse positivamente de los eventuales obstáculos y adversidades que se presenten en el proceso de implementar efectivamente la idea innovadora.

Respecto al rol mediador de la pasión, aunque a la fecha no se dispone de evidencias empíricas que permitan contrastar dicho hallazgo, su intervención como "tercera variable" ha sido subrayado por distintos autores (Bañuelos et al., 2016; Curran et al., 2015; Vallerand, 2015). En efecto, una reciente investigación desarrollada en India (Narang y Bhatnagar, 2017) demostró su intervención en calidad de mediador total en las relaciones entre variables actitudinales y comportamientos proactivos en el trabajo. Asimismo, el rol mediador de la pasión por el trabajo puede ser comprendido a la luz del modelo de ampliación y construcción emocional (Koky Fredrickson, 2013). Según sus postulados, cuando las personas experimentan sentimientos positivos se modifican sus formas de pensamiento y se extienden sus posibilidades de acción, dando lugar a conductas que optimizan los recursos disponibles (Kok y Fredrickson, 2013). En línea con esta teoría podría asumirse, entonces, que el estado emocional positivo y expansivo asociado a la pasión armoniosa propiciaría mayor flexibilidad y apertura mental, favoreciendo así la identificación de oportunidades de mejora y la generación de ideas novedosas. Al mismo tiempo, sería factible asumir que las personas apasionadas hacia su trabajo capitalizarían más efectivamente sus recursos psicológicos, movilizando los cursos de acción necesarios para el logro de las metas propuestas, redireccionando los esfuerzos en caso de ser necesario, y perseverando hasta lograr desarrollar efectivamente la idea innovadora.

Como todo trabajo empírico el presente estudio no está excepto de debilidades. Entre las principales limitaciones de la investigación realizada hay que citar, por ejemplo, la representatividad de la muestra. Al haber sido seleccionada según un 
muestreo por disponibilidad, la posibilidad de extrapolar los resultados fuera de los confines del estudio realizado se encuentra vedada. No obstante, analizando comparativamente la muestra estudiada y la población objetivo surge que la muestra estudiada se aproxima en edad y género a la población docente santafesina en general $y$, rafaelina, en particular. En este sentido, según relevamientos oficiales publicados por el Ministerio de Trabajo de la Provincia de Santa Fe (2017), 8 de cada 10 docentes son mujeres, y la edad promedio oscila entre los 35 y los 39 años. Asimismo, la proporción de docentes que se desempeñan en establecimientos de gestión pública y de gestión privada también se encuentra relativamente bien representada en esta muestra; habida cuenta que en el sistema educativo rafaelino el $87 \%$ de las escuelas de nivel primario son estatales (Ministerio de Trabajo de la Provincia de Santa Fe, 2017).

Una segunda limitación podría estar vinculada al diseño transversal utilizado, el que impide cualquier inferencia de causalidad en sentido estricto entre las variables estudiadas. Frente a este panorama, sería importante que futuras investigaciones consideren la posibilidad de llevar a cabo estudios longitudinales para establecer el orden causal de las relaciones encontradas en el presente trabajo. Otra limitación del estudio podría estar referida al carácter auto descriptivo de las escalas y cuestionarios empleados para la recolección de los datos. Por tratarse de medidas que evalúan comportamientos percibidos, las respuestas podrían verse contaminadas por el componente subjetivo que esto conlleva. Para superar esta limitación sería valioso que futuros estudios evalúen la posibilidad de incluir datos provenientes de otras fuentes, con el propósito de incrementar la validez externa.

Más allá de sus limitaciones, cabe destacar que esta investigación constituye un aporte original a la literatura psicológica al dejar establecida la sinergia entre las diferencias individuales (en términos de recursos psicológicos y actitudes hacia el trabajo), y conductas organizacionalmente deseables (en este caso, el comportamiento innovador). Más aún, si bien la innovación educativa ha recibido un extenso tratamiento por parte de sociólogos, pedagogos y cientistas de la educación, el abordaje psicológico de esta temática es relativamente reciente. Por ende, los hallazgos obtenidos contribuyen a cubrir un área de vacancia, habida cuenta que de la revisión de la literatura más actualizada no surge ningún estudio que haya examinado simultáneamente esta particular combinación de constructos, o que les haga jugar a cada uno los roles que aquí les fueron asignados.

Las principales implicaciones prácticas que podrían derivarse de estos hallazgos se vinculan, en primer lugar, a la necesidad de propiciar ambientes laborales que contribuyan al florecimiento del capital psicológico y la pasión armoniosa. En este sentido, la evidencia muestra consistentemente el CapPsi y la pasión constituyen estados desarrollables y susceptible al cambio (Omar et al., 2014; Carter y Youssef区 Morgan, 2019; Salessi et al., 2017; Vallerand, 2015). A su vez, del presente estudio se desprende que el CapPsi es un importante predictor de la pasión; y que, al mismo tiempo, contar con empleados con alto CapPsi y pasión por su trabajo, allana el 
camino a la innovación. Por lo tanto, invertir en intervenciones destinadas a promover los recursos psicológicos de los trabajadores supondrá para las organizaciones mayores niveles de rendimiento, compromiso y bienestar entre sus miembros; lo que conducirá, a su vez, a desempeños innovadores con impacto directo en la efectividad organizacional.

La agenda para futuros estudios debería orientanse, en primer lugar, a replicar este modelo en otras muestras de trabajadores. Así, por ejemplo, algunas investigaciones podrían extender los límites del universo estudiado en esta oportunidad, analizando otros grupos ocupacionales de modo de corroborar si las particularidades de cada profesión dejan su impronta en las relaciones encontradas. A su vez, estudios transculturales podrían contrastar el modelo desarrollado con miras a iluminar la posible influencia de la cultura en las relaciones encontradas. En última instancia, la verificación del modelo aquí presentado en otros contextos, constituiría un valioso aporte para determinar si, más allá de las diferencias culturales, existen factores psicológicos que trascienden los contextos sociales y políticos.

\section{Agradecimientos}

Este trabajo se produjo en el marco del proyecto "Comportamiento Innovador Docente: Entre el Capital Psico-social y el Bienestar en el Trabajo", financiado por la Universidad Nacional de Rafaela (Res. CS 0042017/18). La autora agradece por el financiamiento y apoyo recibido en la ejecución de este estudio.

\section{Referencias}

Abbas, M. y Raja, U. (2015). Impact of psychological capital on innovative performance and job stress. Canadian Journal of Administrative Sciences, 32(2), 128-138.

APA. (2017). Ethical principles of psychologists and code of conduct. APA. HTTP://WWW.APA.ORG/ ETHICS/CODE/

Ato, M., López, J. J. y Benavente, A. (2013). Un sistema de clasificación de los diseños de investigación en psicología. Anales de Psicología, 29(3), 1038-1059. HTTPS://DOI.ORG/10.6018/ANALESPS.29178511

Bañuelos, A., Descals, F. y Castaño, M. (2016). ¿Podemos apasionarnos por el trabajo? Una revisión de la pasión para el trabajo. Papeles del Psicólogo, 37(3), 165-169.
Carter, J. W. y Youssef-Morgan, C. M. (2019). The positive psychology of mentoring: A longitudinal analysis of psychological capital development and performance in a formal mentoring program. Human Resource Development Quarterly, 30(3), 383-405. HTTPS://DOI.ORG/10.1002/HRDQ.21348

Cheung, G. W. y Wang, C. (2017). Current approaches for assessing convergent and discriminant validity with SEM: Issues and solutions. Academy of Management Proceedings, 2017(1), 12706. HTTPS://DOI.ORG/10.5465/ AMBPP.2017.12706АBSTRACT

CONICET. (2006). Resolución No. 2857: CONICET: Lineamientos para el comportamiento ético en las Ciencias Sociales y Humanidades. CONICET. 
Curran, T., Hill, A., Appleton, P., Vallerand, R. y Standage, M. (2015). The psychology of passion: A meta-analytical review of a decade of research on intrapersonal outcomes. Motivation \& Emotion, 39(5), 631-655. HTTPS://DOI.ORG/10.1007/s11031015-9503

De Spiegelaere, S., Van Gyes, G. y Van Hootegem, G. (2014). The concept of innovative work behaviour: Definition and orientation. Gedrag en Organisatie, 27(2), 139-156.

Dominguez Lara, S. A. (2016). Evaluación de la confiabilidad del constructo mediante el coeficiente $\mathrm{H}$ : Breve revisión conceptual y aplicaciones. Psychologia, 10(2), 94. HTTPS://DOI. ORG/10.21500/19002386.2134

Gulyani, G. y Bhatnagar, J. (2017). Mediator analysis of passion for work in Indian millennials: Relationship between protean career attitude and proactive work behavior. Career Development International, 22(1), 50-69. HTTPS://DOI. ORG/10.1108/CDI-04-2016-0057

Hayes, A. F. (2013). Methodology in the social sciences. Introduction to mediation, moderation, and conditional process analysis: A regressionbased approach. Guilford Press.

Hopwood, C. J. (2007). Moderation and mediation in structural equation modeling: Applications for early intervention research. Journal of Early Intervention, 29(3), 262-272. HTTPS://DOI. ORG/10.1177/105381510702900305

Huang, L. y Luthans, F. (2015). Toward better understanding of the learning goal orientationcreativity relationship: The role of positive psychological capital. Applied Psychology, 64(2), 444-472. HTTPS://DOI.ORG/10.1111/APPS.12028

Kaur, K. y Gupta, V. (2016). The impact of personal characteristics on innovative work behaviour: An exploration into innovation and its determinants amongst teachers. The International Journal of Indian Psychology, 3(11).

Klaeijsen, A., Vermeulen, M. y Martens, R. (2018). Teachers' innovative behaviour: The importance of basic psychological need satisfaction, intrinsic motivation, and occupational self-efficacy. Scandinavian Journal of Educational Research, 62(5), 769-782. HTTPS://DOI.ORG/10.1080/0031383 1.2017 .1306803

Kok, B. E. y Fredrickson, B. L. (2013). Positive emotion: How positive emotions broaden and build. En J. J. Froh y A. C. Parks (Eds.), Activities for teaching positive psychology: A guide for instructors (pp. 61-63). American Psychological Association.
Luthans, F y Youssef-Morgan, C. M. (2017). Psychological capital: An evidence-based positive approach. Annual Review of Organizational Psychology and Organizational Behavior, 4(1), 339-366. HTTPS://DOI.ORG/10.1146/ANNUREVORGPSYCH-032516-113324

Madrid, H. P., Diaz, M. T., Leka, S., Leiva, P. I. y Barros, E. (2018). A finer grained approach to psychological capital and work performance. Journal of Business and Psychology, 33(4), 461477. HTTPS://DOI.ORG/10.1007/S10869-017-9503-Z

Messmann, G., Stoffers, J., Van der Heijden, B. y Mulder, R. H. (2017). Joint effects of job demands and job resources on vocational teachers' innovative work behavior. Personnel Review, 46(8), 1948-1961. HTTPS://DOI.ORG/10.1108/PR03-2016-0053

Ministerio de Trabajo de la Provincia de Santa Fe. (2017). Boletín 34: Composición y evolución del empleo registrado. Ministerio de Trabajo de la Provincia de Santa Fe. HTTPs://www.SAnTAFE. GOV.AR/INDEX.PHP/CONTENT/VIEW/FULL/93760

Montani, F., Odoardi, C. y Battistelli, A. (2014). Individual and contextual determinants of innovative work behaviour: Proactive goal generation matters. Journal of Occupational and Organizational Psychology, 87(4), 645-670. HTTPS://DOI.ORG/10.1111/JOOP.12066

Oeij, P., Rus, D. y Pot, F. D. (Eds.). (2017). Workplace innovation: Theory, research and practice. Springer.

Omar, A., Salessi, S. y Urteaga, F. (2014). Diseño y validación de una nueva escala para medir capital psicológico. Liberabit, 20(2), 315-323.

Podsakoff, P. M., MacKenzie, S. B. y Podsakoff, N. P. (2012). Sources of method bias in social science research and recommendations on how to control it. Annual Review of Psychology, 63(1), 539-569. HTTPS://DOI.ORG/10.1146/ANNUREVPSYCH-120710-100452

Salessi, S. (en prensa). Comportamiento innovador en el trabajo: Análisis factorial confirmatorio de la escala de Janssen. Interdisciplinaria. Revista de Psicología y Ciencias Afines.

Salessi, S. (en prensa). Innovative work behaviour: Development and validation of a scale for teachers. Revista Interamericana de Psicología.

Salessi, S. y Omar, A. (en prensa). Pasión por el trabajo: Un estudio instrumental con trabajadores argentinos. Psykhe.

Salessi, S. y Omar, A. (2017). Comportamientos proactivos en el trabajo: Una puesta al día. Revista Argentina de Ciencias del Comportamiento, 9(3), 82-103. 
Salessi, S., Omar, A. y Vaamonde, J. D. (2017) Conceptual considerations of work passion. Ciencias Psicológicas, 11(2), 165-178.

Sameer, Y. M. (2018). Innovative behavior and psychological capital: Does positivity make any difference? Journal of Economics and Management, 32, 75-101. HTTPS://DOI. ORG/10.22367/JEM.2018.32.06

Sun, Y. y Huang, J. (2019). Psychological capital and innovative behavior: Mediating effect of psychological safety. Social Behavior and Personality: An International Journal, 47(9), 1-7. HTTPS://DOI.ORG/10.2224/SBP.8204

Tabachnick, B. y Fidell, L. S. (2013). Using multivariate statistics. Pearson.

Taştan, S. (2016). Psychological capital: A positive psychological resource and its relationship with creative performance behavior. Anadolu. University Journal of Social Sciences, 16(2), 101118. HTTPS://DOI.ORG/10.18037/AUSBD.389199

Thurlings, M., Evers, A. T. y Vermeulen, M (2015). Toward a model of explaining teachers' innovative behavior: A literature review. Review of Educational Research, 85(3), 430-471. HTTPS:// DOI.ORG/10.3102/0034654314557949
UNESCO. (2016). Innovación educativa. Organización de las Naciones Unidas para la Educación, la Ciencias y la Cultura.

Vallerand, R. (2015). The psychology of passion. A dualistic model. Oxford University Press.

Vu, P., Fredrickson, S. y Moore, C. (Eds.). (2017). Handbook of research on innovative pedagogies and technologies for online learning in higher education. IGI Global. HTTPs://DOI. ORG/10.4018/978-1-5225-1851-8

Wang, X.-H. F., Fang, Y., Qureshi, I. y Janssen, O. (2015). Understanding employee innovative behavior: Integrating the social network and leader-member exchange perspectives. Journal of Organizational Behavior, 36(3), 403-420. HTTPs:// DOI.ORG/10.1002/JOB.1994 